A total of 35 patients had a positive past psychiatric history, out of which 17 were on maintenance psychotropic medications. Insomnia was the commonest psychiatric symptom $(n=65)$, followed by anxiety $(n=52)$, agitation $(n=42)$, depression $(n=39)$, changes in appetite $(n=32)$ and irritability $(n=30)$. The principal psychiatric diagnoses made were delirium $(n=29)$, acute stress reaction or adjustment disorder $(n=25)$, depression $(n=16)$, mania $(n=15)$, anxiety $(n=14)$, non-affective psychosis $(n=13)$, and dementia $(n=6)$. Approximately half of the patients with mania or non-affective psychosis had it as their first-onset disorder. Conclusion. SARS-CoV-2, in both symptomatic and asymptomatic patients, is associated with a wide range of psychiatric morbidity which emphasizes clinicians' vigilance for psychiatric symptoms. Insomnia was the commonest neuropsychiatric symptom which may have clinical practice and potential preventive strategies implications.

Delirium, the commonest diagnosis in the study carries high morbidity and mortality and may reflect SARS-Cov-2 propensity to affect the brain directly and indirectly through a cytokine storm, organ failure, and prothrombotic state. Patients can also present with new-onset mania or non-affective psychosis. It is noteworthy that about two-thirds of the patients had no past psychiatric history.

This study, along with expanding body of evidence may assist with resource allocation and liaison psychiatry services planning. It also underscores the importance of designing future studies to better understand longer-term psychiatric sequelae of COVID-19.

\section{Impact of COVID-19 on the mental health of healthcare workers in different regions of the world}

Mahfuja Islam ${ }^{1 \star}$, Philip George ${ }^{2}$, Sindhu Sankaran ${ }^{3}$, Janet Leu Su Hui ${ }^{2}$ and Tzun Kit ${ }^{2}$

${ }^{1}$ Ashford and St Peter's NHS Foundation Trust; ${ }^{2}$ International Medical University and ${ }^{3}$ All India Institute of Medical Sciences ${ }^{\star}$ Corresponding author.

\section{doi: 10.1192/bjo.2021.689}

Aims. The global health system is facing a serious challenge after the recent outbreak of COVID-19 coronavirus infection which was first identified in Wuhan, China in November 2019 and declared as a pandemic in March 2020 by WHO. There is a wide consensus that this pandemic has negative psychosocial consequences as well as unforeseeable provision of mental health care services and just not on physical health alone. The aim of this research study is to determine the prevalence of psychological distress and to identify the sociodemographic variables with the main attributable factors associated with the psychological distress among healthcare workers and suggestions on how to reduce the impact on the mental health of healthcare workers during the COVID-19 pandemic in different regions of the world.

Method. We performed a cross-sectional study from September-November 2020. We used a self-administered survey tool which was distributed electronically to healthcare workers across the globe. The data were stored on an online database with password protected devices where survey responses were restricted to investigators exclusively.

Data collected were: 1) Socio-demographic data (age, gender, marital status, ethnicity, religion, role in the healthcare, region of practice); 2) Psychological General Well-Being Index (PGWBI) questionnaire which contains 22 standardized items. This is a subjective assessment to score the degree of psychological well-being by focusing on 6 domains: depression; anxiety; positive-well-being; self-control; vitality and general health; 3)
Subjective assessment from respondents of the main attributable factors causing psychological distress and suggested methods to help reduce the impact on mental health on health care workers.

Result. Majority out of the 217 respondents were from a younger age group; females and married/domestic partnership, mainly from Western Pacific Region, South East Asian and the African Region. More than half the respondents were moderate-severely psychologically distressed and the three main attributable factors causing psychological distress were: fear of family/friends contracting COVID-19 followed by lack of PPE and discomfort caused by wearing PPE for long hours. Respondents suggested that the distress would be reduced if: more resources were provided in hospital; protocols and guidelines were implemented and counselling facilities with recreational activities were available to frontline workers.

Conclusion. This study showed that the COVID-19 pandemic has affected the mental health of healthcare workers and more support or strategies need to come in place to protect frontline workers at the time of crises.

\section{Impact of COVID-19 on the mental health of healthcare workers in different regions of the world}

Mahfuja Islam ${ }^{1 \star}$, Philip George ${ }^{2}$, Sindhu Sankaran³, Janet Leu Su $\mathrm{Hui}^{2}$ and Tzun $\mathrm{Kit}^{2}$

${ }^{1}$ Ashford and St Peter's NHS Foundation Trust; ${ }^{2}$ International Medical University and ${ }^{3}$ All India Institute of Medical Sciences ${ }^{\star}$ Corresponding author.

doi: 10.1192/bjo.2021.690

Aims. The global health system is facing a serious challenge after the recent outbreak of COVID-19 coronavirus infection which was first identified in Wuhan, China in November 2019 and declared as a pandemic in March 2020 by WHO. There is a wide consensus that this pandemic has negative psychosocial consequences as well as unforeseeable provision of mental health care services and just not on physical health alone. The aim of this research study is to determine the prevalence of psychological distress and to identify the sociodemographic variables with the main attributable factors associated with the psychological distress among healthcare workers and suggestions on how to reduce the impact on the mental health of healthcare workers during the COVID-19 pandemic in different regions of the world.

Method. We performed a cross-sectional study from September-November 2020. We used a self-administered survey tool which was distributed electronically to healthcare workers across the globe. The data were stored on an online database with password protected devices where survey responses were restricted to investigators exclusively.

Data collected were: 1) Socio-demographic data (age, gender, marital status, ethnicity, religion, role in the healthcare, region of practice); 2) Psychological General Well-Being Index (PGWBI) questionnaire which contains 22 standardized items. This is a subjective assessment to score the degree of psychological well-being by focusing on 6 domains: depression; anxiety; positive-well-being; self-control; vitality and general health; 3) Subjective assessment from respondents of the main attributable factors causing psychological distress and suggested methods to help reduce the impact on mental health on health care workers. Result. Majority out of the 217 respondents were from a younger age group; females and married/domestic partnership, mainly from Western Pacific Region, South East Asian and the African Region. More than half the respondents were moderate-severely psychologically distressed and the three main attributable factors 
causing psychological distress were: fear of family/friends contracting COVID-19 followed by lack of PPE and discomfort caused by wearing PPE for long hours. Respondents suggested that the distress would be reduced if: more resources were provided in hospital; protocols and guidelines were implemented and counselling facilities with recreational activities were available to frontline workers.

Conclusion. This study showed that the COVID-19 pandemic has affected the mental health of healthcare workers and more support or strategies need to come in place to protect frontline workers at the time of crises.

\section{A survey of Irish psychiatric trainees attitudes to balint groups \\ Sudha Jain ${ }^{1 \star}$, Emma Adams ${ }^{2}$ and Alyson Lee ${ }^{1}$ \\ ${ }^{1}$ Mid East Kildare West/ Wicklow Menatal Health Services and \\ ${ }^{2}$ Kildare West Wicklow Mental Healt Services \\ ${ }^{\star}$ Corresponding author.}

doi: 10.1192/bjo.2021.691

Aims. 1. To compare the experience of psychiatric trainees in Ireland of online Balint Groups (BG) in contrast to face to face groups.

2. To assess the general attitudes of trainees to BG using the Psychological Medical Inventory (PMI) (Ireton and Sherman, 1988) scale.

Method. An online survey comprising two sections: 1. A questionnaire exploring participants experience, effectiveness and obstacles to attending the two formats of BG with a free text box response. 2. General attitude of trainees towards BG using PMI scale.

An online cross-sectional survey using Survey Monkey. An invitation to participate in the survey was emailed to all trainees by the College of Psychiatrists in Ireland. All data were anonymised, and all data processing was conducted in line with GDPR. Statistical analysis was undertaken using Microsoft Excel. Thematic analysis was applied to the free-text box responses.

Result. 16.49\% (64/388) responded to the survey. Responses were uniform across all stages of training. $97 \%$ of respondents attended BG; $72 \%$ attended both formats, $25 \%$ attended only face-to-face and $3 \%$ online only. $65 \%$ of respondents preferred face to face compared to $18 \%$ online, whilst $11 \%$ stated no preference.

On thematic analysis, trainees asserted a preference for face-to-face, describing better group cohesion, feeling safer to share, increased ease of interpreting non-verbal communication, and that conversation was more fluid. They described greater ease of engagement with the group/facilitator and preferred direct social interaction with peers.

Conversely, most trainees acknowledged that online groups were convenient to attend, less time consuming \& mitigated COVID risk associated with face-to-face meetings. Common themes against the use of online groups were: less psychotherapeutic in nature, technical issues, silences, unable to see participants faces and as though speaking "into the void".

Regarding trainees' attitudes to attending BG, most of the trainees found BG had been beneficial in developing more interest and confidence in dealing with the psychological aspects of patient care. Trainees agreed that skills improved in developing an excellent doctor-patient relationship, recognising patients under stress/ in distress, systemically obtaining psychological information and making treatment decisions based upon psychological needs and psychotherapeutic engagement. They agreed that they could better understand the influence of doctors' emotions on the doctor-patient relationship.

Conclusion. This survey showed that most trainees find BG beneficial in developing better doctor-patient relationships, preferring face-to-face rather than online BG. However, they found online more convenient. A blended learning approach could provide trainees with the benefits of both formats of BG.

A decade of community-based participatory research: from a tentative start to a fruitful approach in the field of mental health: a scoping review

Aoife M. Janmohamed

Maastricht University

doi: 10.1192/bjo.2021.692

Aims. This scoping review aimed to identify and analyse studies utilising Community-Based Participatory Research (CBPR) to design and/or disseminate a mental health $(\mathrm{MH})$ intervention with underserved communities around the globe. This was with the intention of updating the knowledge base on this area, and identifying both areas of promise in this field as well as any gaps for future work to fill.

Method. This scoping review was conducted using the Joanna Briggs Institute's Scoping Review Manual and the Preferred Reporting Items for Systematic reviews and Meta-Analyses extension for Scoping Reviews (PRISMA-ScR) checklist. Three databases, Scopus, PubMed and Sage Journals, were searched to identify relevant studies, using the three terms 'CBPR', 'underserved' and 'mental health', and words to the equivalent.

Result. The search identified 607 English-language sources published between 1st January 2010 and 30th June 2020. Following duplicate removal, screening and bibliography scanning, 34 highly relevant studies remained. The studies were varied in their chosen context, $\mathrm{MH}$ gap, how they gave meaning to the participatory approach, how they defined their successes, and what strengths and challenges were encountered in CBPR's application to this field. Briefly, all bar one of the studies were focused on underserved communities within high-income countries (HICs); many focused solely on women and youth groups; and finally, the use of technology and talking therapies were noted to achieve particular success.

Conclusion. CBPR is commonly used to engage the underserved through long-term partnership building and equitable stakeholder involvement, shifting the dialogue from research on to research with communities. This unique, needs-oriented approach harbours mutual ownership of the research, empowering historically disenfranchised individuals to become actively involved in reducing identified health disparities. In the field of $\mathrm{MH}$, this is of great importance and need in many underserved communities due to issues of access, heightened by a distrust in mainstream services as well as by the stigma attached to MH conditions.

As compared to studies in this field ten years ago, CBPR has become much more established, with this review noting a remarkable increase in $\mathrm{MH}$ projects utilising this approach. Furthermore, the addition of new technologies to this field was shown to offer significant promise in overcoming access barriers, hoping to ultimately narrow identified $\mathrm{MH}$ gaps. Nonetheless, further work on the prevailing gender and HIC biases, and for a review including relevant Spanish-language studies, are still required in order to form a more global overview of this field. 